\title{
Light element opacities of astrophysical interest from ATOMIC
}

\author{
J. Colgan ${ }^{1}$, D. P. Kilcrease ${ }^{1}$, N. H. Magee, Jr. ${ }^{1}$, \\ J. Abdallah, Jr. ${ }^{1}$, M. E. Sherrill ${ }^{1}$, \\ C. J. Fontes ${ }^{2}$, P. Hakel ${ }^{2}$, and H. L. Zhang ${ }^{2}$ \\ ${ }^{1}$ Theoretical Division, Los Alamos National Laboratory, Los Alamos, NM 87545 \\ ${ }^{2}$ Computational Physics Division, Los Alamos National Laboratory, Los Alamos, \\ $N M 87545$
}

\begin{abstract}
We report on our continued work to produce a new set of local-thermodynamicequilibrium (LTE) opacity tables using the Los Alamos ATOMIC code. OPLIB tables for the 30 elements from $\mathrm{H}$ through $\mathrm{Zn}$ are almost complete and are expected to be available in 2015. In this paper we present some examples of our new opacities from ATOMIC. We provide comparisons of our opacities with a series of opacity measurements that were performed on $\mathrm{Fe}$ and $\mathrm{Al}$ some years ago. We also present opacities at conditions of interest to stellar modeling and compare and contrast the opacity of various transition metals with OP calculations that are commonly used in astrophysical modeling.
\end{abstract}




\section{Introduction}

In recent years efforts have been underway at Los Alamos National Laboratory (LANL) to generate a new set of opacity tables for the 30 elements hydrogen through zinc. Although the OPLIB database maintained by LANL contains opacity tables generated using the LEDCOP code [1], the last of these were computed more than 10 years ago. The decision was made that new opacity tables were desirable, due to improved physics capabilities in generating equations-of-state [2,3] and much more powerful computing resources, which allow significantly more detailed atomic structure calculations than were possible 10 or more years ago. These new tables are being prepared using the modern ATOMIC opacity and plasma kinetics code $[4,5]$. Recent progress towards our goal of new tables has been previously reported [6,7], and this manuscript provides more examples of the opacities that will be available in our new dataset.

Other comprehensive opacity datasets have been publicly available for some time, in particular from the Opacity Project (OP) [8-11] and the Lawrence Livermore National Laboratory (LLNL) OPAL [12] databases. Although these opacity tables show, for the most part, good agreement with each other and have helped the progression of stellar modeling, recent work has cast doubt on some of the consistency and/or quality of the opacities within these tables [1316]. These studies have added fresh impetus to the need for modern opacity calculations, and several other large-scale opacity efforts are also underway [17-19].

We aim to eventually supersede all of the opacity data in the Los Alamos OPLIB database generated from the legacy LEDCOP calculations, the last of which were made over 10 years ago, with data from our new ATOMIC calculations. The new data will eventually be made available through our opacity website [20]. In the following section we provide a brief theoretical description of the calculations used to generate our new opacities and follow that with several examples of selected monochromatic opacities from ATOMIC. We end with a short conclusion.

\section{Theoretical Developments}

The major aspects of how opacities are computed within ATOMIC have been previously discussed by us $[6,7]$. Opacities from ATOMIC have also been recently used to compare with experiments on Fe [21] at conditions near the base of the solar convection zone and $\mathrm{Fe}$ and $\mathrm{Ni}[18,22]$ at conditions relevant to stellar atmospheres. The major theoretical development in our opac- 
ity calculations since our previous report [7] has been the improvement of our equation-of-state (EOS) package, known as ChemEOS [2].

The ChemEOS model is based on the minimization of the free energy in the chemical picture [2]. It was recently found that this model produced unphysical behavior in the pressure and average ionization trends for some temperatures and densities in Fe. This was traced to the ion-electron contribution to the free energy [23]. A modification to this term was thus introduced that ensures that electron-ion binding is more consistently taken into account and ensures that the correct weak-coupling limit is reached. This development is discussed in detail in a related submission to these proceedings [3].

Apart from this modification, our opacity calculations proceed as discussed previously [6,7]. The Los Alamos semi-relativistic CATS atomic structure code [24] (based on Cowan's codes [25]) is used to compute large atomic datasets. The GIPPER code [26] provides photoionization cross sections. These codes compute data that are read into the ATOMIC code [4,5] and used, along with the EOS computed using the same dataset, to produce opacities for wide ranges of temperatures and densities.

For the transition metal opacities presented here, we used a mixed-UTA (MUTA) approach [27]. The MUTA method retains all of the strongest lines in a given transition array, which allows an accurate spectral description to be generated from a configuration-average set of populations. This approach allows single-configuration fine-structure detail to be included in a relatively computationally inexpensive manner. Calculations that use level-resolved populations as well as configuration-interaction are too computationally intensive at present. In the calculations presented here, we retained all lines for transition arrays that contained less than $10^{5}$ lines within the array. We have found that, for almost all conditions of interest, this parameter choice allows essentially all lines of importance to be explicitly included in the bound-bound opacity contribution. The MUTA approach has been compared to previous Fe transmission measurements, where excellent agreement was obtained [28] for a temperature of $160 \mathrm{eV}$ and an electron density of a few times $10^{21} \mathrm{~cm}^{-3}$.

\section{Results}

In previous reports [6,7], we compared our new mean opacity calculations for $\mathrm{H}, \mathrm{He}, \mathrm{C}$ and $\mathrm{O}$ with $\mathrm{OP}$ and $\mathrm{OPAL}$ calculations. In the following section we provide comparisons of our new monochromatic opacities of $\mathrm{Al}$ and $\mathrm{Fe}$ with various opacity measurements that were made in the 1990s [29-32]. We then present some monochromatic opacities for transition metals of interest to astrophysical modeling of stellar atmospheres. 


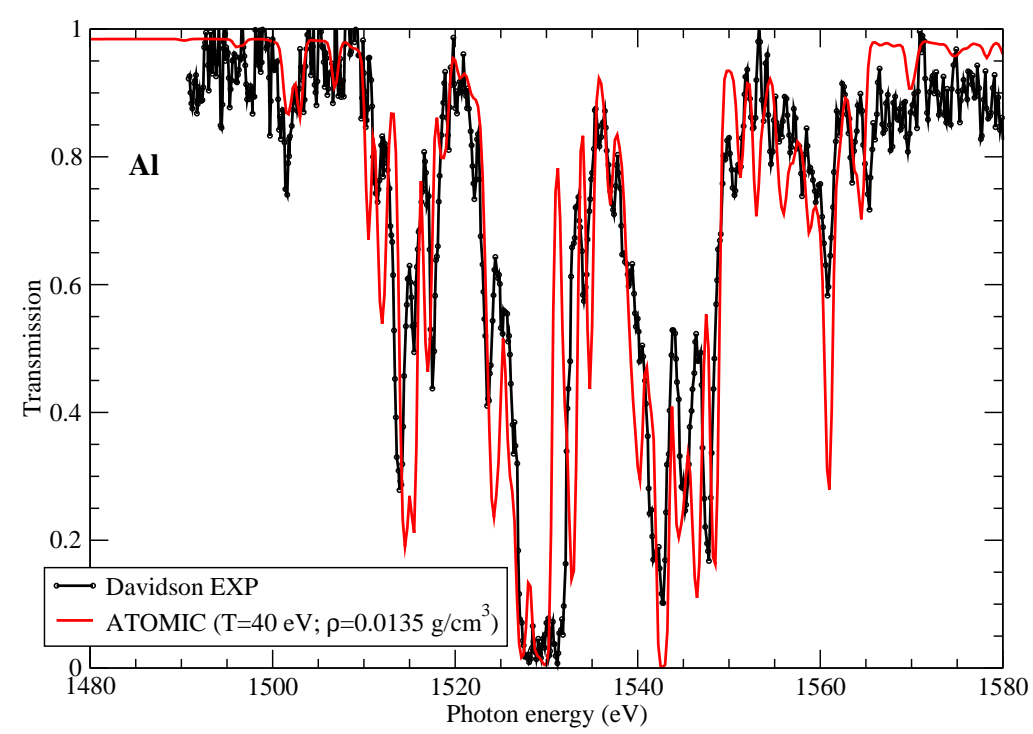

Fig. 1. Measured transmission spectrum of Al (black line) [29] compared with an ATOMIC calculation at a temperature of $40 \mathrm{eV}$ and a mass density of $0.0135 \mathrm{~g} / \mathrm{cm}^{3}$.

\subsection{Comparison with opacity measurements}

In Fig. 1 we compare our ATOMIC calculations for the opacity of Al with the measured transmission of Ref. [29]. A previous Los Alamos calculation [33] was in very good agreement with this measurement, although that one-off calculation did not form part of the Los Alamos opacity tabular data at that time. We compare the measured transmission with an ATOMIC calculation at a temperature of $40 \mathrm{eV}$ and mass density of $0.0135 \mathrm{~g} / \mathrm{cm}^{3}$, which were the conditions found in Ref. [33] that compared best to the measurement. We find that the ATOMIC calculation is in good agreement with the measured transmission, reproducing all the main features found experimentally. Most of the measured features are due to $1 s-2 p$ inner-shell transitions in ions of $\mathrm{Al}$ from $\mathrm{Al}^{5+}$ to $\mathrm{Al}^{9+}$. Some of the finer details are not in perfect agreement. The previous Los Alamos calculation, which used atomic structure that included full configuration-interaction (CI), are in slightly better agreement than the results presented in Fig. 1. This is likely due to the effects of full configuration-interaction, since we recall that the calculated opacity of Al using ATOMIC at these conditions relies on atomic structure calculations that include intermediate-coupling, not full CI. However, we emphasize that the ATOMIC calculations presented in Fig. 1 are the same as used for our new opacity tables, for which full CI calculations are not practical for the large numbers of configurations that need to be included in such calculations. 


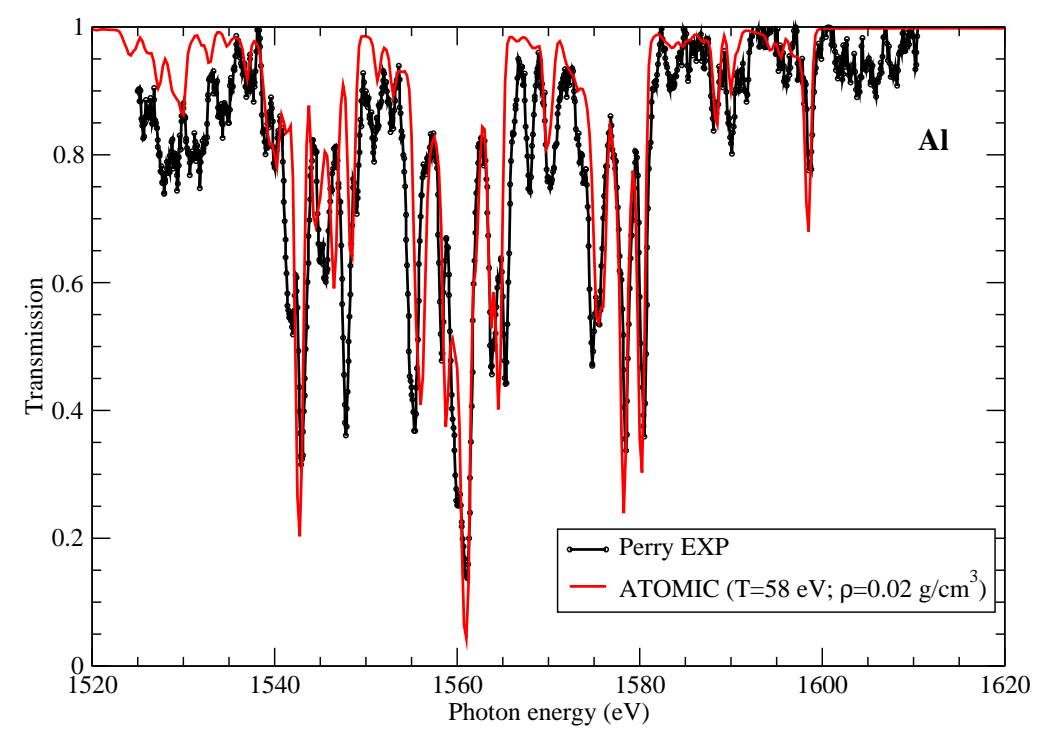

Fig. 2. Measured transmission spectrum of Al (black line) [30] compared with an ATOMIC calculation at a temperature of $58 \mathrm{eV}$ and a mass density of $0.02 \mathrm{~g} / \mathrm{cm}^{3}$.

In Fig. 2 we show an $\mathrm{Al}$ transmission measurement [30] concerning a plasma at a somewhat higher temperature of $58 \mathrm{eV}$. This spectrum, which is over a slightly higher photon energy range, is also in good agreement with our ATOMIC calculation at the conditions indicated in Fig. 2. Again, most of the inner-shell $1 s-2 p$ spectral features are reproduced by the calculation, with the exception of the narrow and strong feature at around a photon energy of $1545 \mathrm{eV}$. Several of the other prominent features (for example the line at $1555 \mathrm{eV}$ ) in the calculation are at slightly different photon energies than were found experimentally (with a difference between theory and experiment of about $1 \mathrm{eV}$ ), which again is likely due to the omission of full CI effects in the current ATOMIC calculations.

We now turn to comparisons of transmission measurements made on Fe plasmas with our ATOMIC calculations. Figure 3 shows a measurement [31] that was made on an iron target mixed with sodium fluoride using the older NOVA laser at Lawrence Livermore National Laboratory. The ATOMIC calculation shown in Fig. 3 is the result of the opacity of Fe added to the opacity of $\mathrm{Na}$, each computed at the appropriate mass densities as stated in Ref. [31]. Addition of the F opacity to the calculation did not change the resulting transmission significantly, and we omit this contribution because of uncertainty as to the $\mathrm{F}$ density in the center of the target, and because the Fe and NaF were not uniformly mixed in the plasma. The agreement between the measured transmission and the ATOMIC calculation is quite good, with all the main 


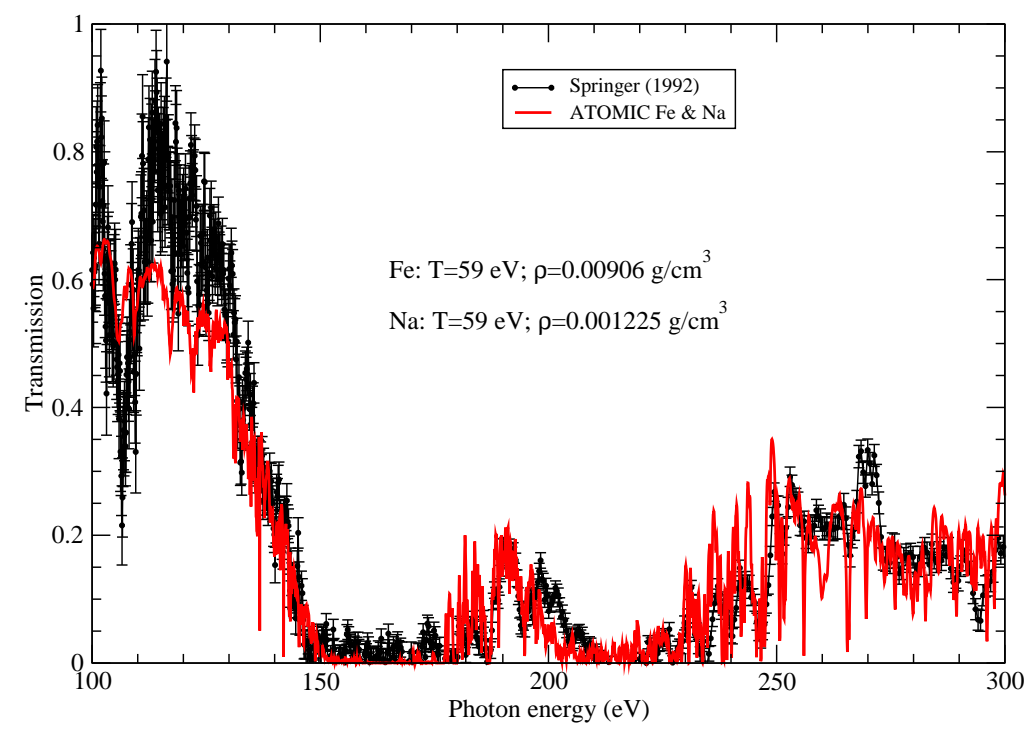

Fig. 3. Measured transmission spectrum of Fe (black line) [31] compared with an ATOMIC calculation at a temperature of $59 \mathrm{eV}$ and a mass density of 0.00906 $\mathrm{g} / \mathrm{cm}^{3}$. We have also added the opacity of $\mathrm{Na}$ to the calculated transmission at the conditions indicated.

features reproduced by the calculation. There is some disagreement in the depth of the feature at $105 \mathrm{eV}$, and in the background transmission at around $115 \mathrm{eV}$, but in all other portions of the spectrum the measurement and theory are in good agreement. The publication of Ref. [31] included comparisons to OPAL calculations and by inspection our new ATOMIC calculations appear to be in good agreement with the OPAL calculations presented in Ref. [31].

In Fig. 4 we examine the transmission spectrum of $\mathrm{Fe}$ at a lower temperature of around $24 \mathrm{eV}$, made using the ASTERIX IV laser in Germany [32]. This study measured the transmission from Al, Fe, and Ho targets, but for brevity we only compare to the Fe measurements here. Since a small spatial temperature gradient of $2 \mathrm{eV}$ was inferred from hydrodynamic simulations of this experiment, we present ATOMIC calculations at both 22 and $24 \mathrm{eV}$, following the comparisons that were published. This relatively small temperature change can lead to significant changes in the opacity at these conditions. We find that both ATOMIC calculations are in reasonable agreement with the measured transmission, with the calculation at $24 \mathrm{eV}$ in slightly closer agreement. The only large difference between the measured transmission and the calculation is found at the lowest photon energies around $75 \mathrm{eV}$, where large differences were also observed between the measurement and the OPAL calculations presented in Ref. [32]. 


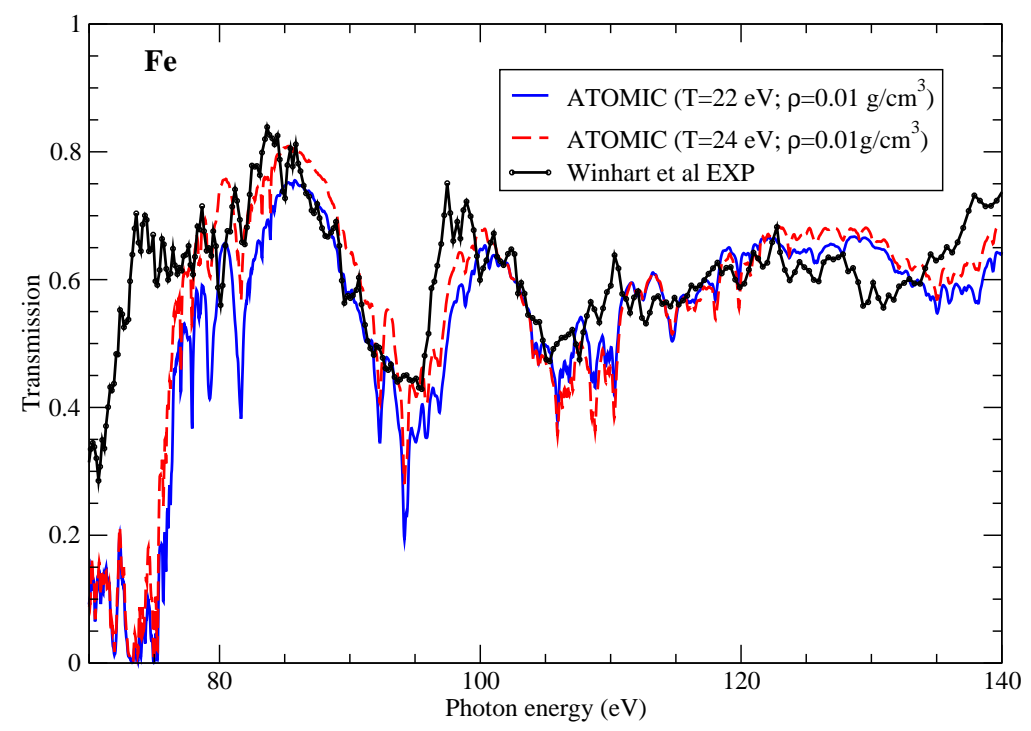

Fig. 4. Measured transmission spectrum of Fe (black line) [32] compared with ATOMIC calculations at temperatures of $22 \mathrm{eV}$ and $24 \mathrm{eV}$ as indicated, both at a mass density of $0.01 \mathrm{~g} / \mathrm{cm}^{3}$.

We end this section by noting several other measurements of Fe transmission spectra $[34,35]$. The measurements of Ref. [34] produced a plasma at a temperature of around $25 \mathrm{eV}$ and a mass density of $8 \mathrm{mg} / \mathrm{cm}^{3}$. Although the agreement between our ATOMIC calculations and the measured data is reasonable, possible contamination of the measured transmission by self-emission makes a detailed comparison difficult. More recent measurements were made from a Fe plasma at a temperature of around $72 \mathrm{eV}$ [35]. A broad photon energy range was measured (150-1200 eV), and it appears that our ATOMIC calculations are in good agreement with the measured transmission. However, the data are not available in numerical form so that a detailed comparison is again difficult to make.

\subsection{Additional Transition metal opacities}

We now turn to an examination of the opacity from additional transition metals of astrophysical interest. Our new opacity tables for $\mathrm{Cr}, \mathrm{Fe}, \mathrm{Ni}$, and $\mathrm{Cu}$ are almost complete, and we provide the monochromatic opacity from each of these in Fig. 5, at a temperature of $15.3 \mathrm{eV}$ and a mass density of $5.48 \times 10^{-3}$ $\mathrm{g} / \mathrm{cm}^{3}$. These conditions are chosen as they approximately reproduce the ionization balance of such elements at conditions found in stellar envelopes and also allow straightforward comparison with OP data [11], since these con- 
ditions are one of the grid points used in the OP opacity tabulation. The opacities of $\mathrm{Cr}, \mathrm{Fe}$, and $\mathrm{Ni}$ extracted from the OP tables [11] are presented in Fig. 6 for comparison purposes. Our earlier work [6] compared the opacity of Fe computed from OP with that computed from ATOMIC at these conditions. Significant differences were found in the monochromatic opacity and resulting Rosseland mean opacity. Although the inclusion of configuration-interaction in the OP calculation is responsible for some of the differences in the opacity at photon energies around $50 \mathrm{eV}[18,22]$, the larger differences between the ATOMIC and OP opacities at higher photon energies imply that differences also exist in the bound-free background opacity. We speculate that this difference may be due to omission of important configurations that represent $3 \mathrm{p}$ to $3 \mathrm{~d}$ promotions in the OP calculations for Fe ions from around Cr-like to Klike. This assertion is supported by the closer agreement between the OP and ATOMIC calculations at larger temperatures (not shown), where the average ionization has moved beyond Ar-like Fe. We also recall that limited (in terms of number of configurations included in the structure calculations) ATOMIC calculations that include full configuration-interaction [22] produced an opacity that was almost identical to the ATOMIC calculations shown in Fig 5 at photon energies above $120 \mathrm{eV}$, at which energy the differences between the OP calculations and all the ATOMIC calculations become significant [6].

The opacity of $\mathrm{Ni}$ as obtained from the OP tables [11] has also been questioned $[18,22]$, since the $\mathrm{OP} \mathrm{Ni}$ opacities at temperatures around $20 \mathrm{eV}$ show large differences with more recent calculations [18]. This finding is perhaps not too surprising, since the OP calculations for Ni used atomic data that were scaled from ab-initio $\mathrm{Fe}$ calculations, i.e. the atomic data used for $\mathrm{Ni}$ was not abinitio. This approach was justified in the earlier OP reports [8] because of the low abundance of $\mathrm{Ni}$ in the Sun. However, for other systems where Ni may be more abundant, the scaling approach could lead to large inaccuracies.

Returning to Fig. 5, we find that the opacities of $\mathrm{Cr}, \mathrm{Fe}, \mathrm{Ni}$, and $\mathrm{Cu}$ show systematic trends. At the same temperature and mass density, one finds that the degree of ionization of each element is the same, around 5.3. The dominant bound-bound feature observed in $\mathrm{Cr}$ at a photon energy around $60 \mathrm{eV}$ moves steadily to higher photon energies as the $\mathrm{Z}$ increases. The intensity of this 'bump' also systematically decreases. Since this feature moves away from the peak of the Rosseland weighting function, the resulting Rosseland mean opacity also steadily decreases with increasing Z. At higher photon energies, we find that the background bound-free (arising from the M-shell) contribution to the total opacity increases with $\mathrm{Z}$. This may be due to the greater number of M-shell electrons that are available to be photoionized at higher $\mathrm{Z}$ values, since the dominant ion moves from K-like $\left(3 d^{1}\right)$ (for $\mathrm{Cr}$ ) to Cr-like $\left(3 d^{6}\right)$ (for $\mathrm{Cu}$ ).

The OP opacities for Cr, Fe, and Ni in Fig. 6 do not however exhibit the trends 


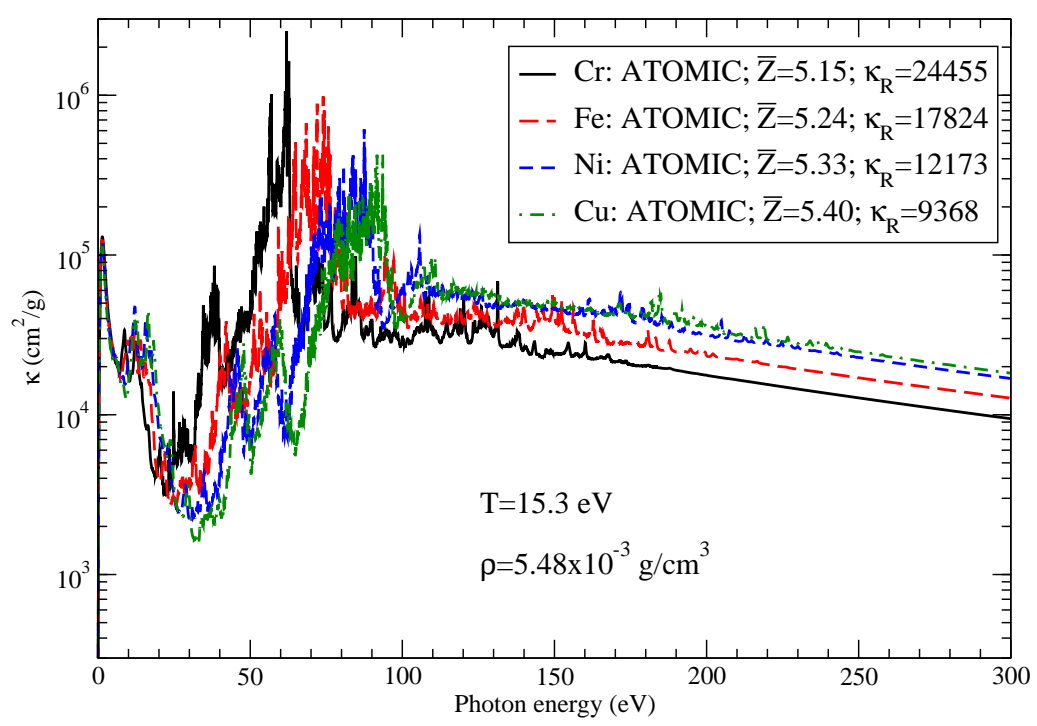

Fig. 5. Monochromatic opacities of $\mathrm{Cr}, \mathrm{Fe}, \mathrm{Ni}$, and $\mathrm{Cu}$ at a temperature of $15.3 \mathrm{eV}$ and a mass density of $5.48 \times 10^{-3} \mathrm{~g} / \mathrm{cm}^{3}$ obtained using the ATOMIC code. The average ionization $(\bar{Z})$ and Rosseland mean opacity (in $\mathrm{cm}^{2} / \mathrm{g}$ ) are indicated for each case in the figure caption.

found in the ATOMIC opacities. As we move from $\mathrm{Cr}$ to Fe, the large boundbound feature does move to higher photon energies, but the bound-free opacity at higher photon energies decreases, in contradiction to the trends found in the ATOMIC opacities in Fig. 5. Furthermore, the opacity of $\mathrm{Ni}$ at photon energies above $150 \mathrm{eV}$ appears very similar to that of $\mathrm{Fe}$, again in contradiction with the trends found in the ATOMIC opacities. We note that the OP calculations of Cr opacity also used scaled atomic data from Fe [8]. This approximation does not appear to be robust and casts doubt on the single-element opacities from $\mathrm{Cr}$ and $\mathrm{Ni}$ from the $\mathrm{OP}$ database. Finally we note that, although the Rosseland mean opacity of $\mathrm{Cr}$ from the $\mathrm{OP}$ data is reasonably close to the Rosseland mean opacity of Cr from ATOMIC, there are larger (factors of two) differences in the mean opacity between the two methods for $\mathrm{Fe}$ and $\mathrm{Ni}$.

\section{Conclusion}

In this paper we have described some of the new opacities that were produced using the LANL ATOMIC code, following on from our earlier work $[6,7]$. We have compared our new opacities with a selection of transmission measurements from various absorption measurements [29-32] made in efforts 


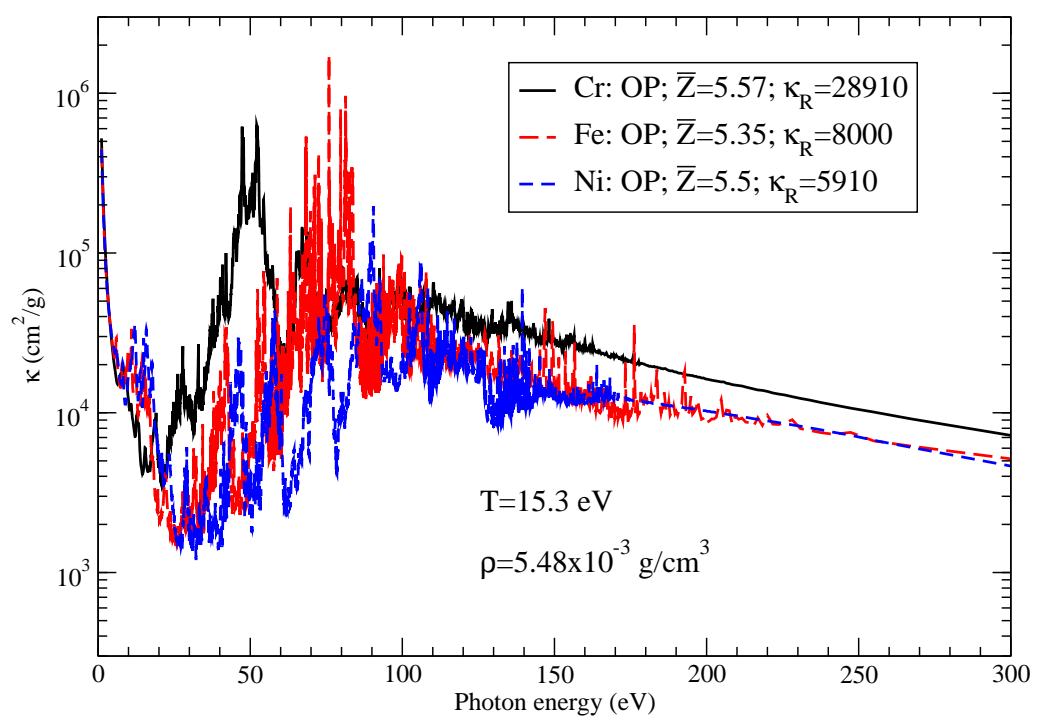

Fig. 6. Monochromatic opacities of $\mathrm{Cr}, \mathrm{Fe}$, and $\mathrm{Ni}$, at a temperature of $15.3 \mathrm{eV}$ and a mass density of $5.48 \times 10^{-3} \mathrm{~g} / \mathrm{cm}^{3}$ obtained using the OP code. The average ionization $(\bar{Z})$ and Rosseland mean opacity (in $\mathrm{cm}^{2} / \mathrm{g}$ ) are indicated for each case in the figure caption.

to benchmark opacity calculations. Overall, the reasonable agreement with these measurements implies a partial validation of our new opacity calculations. We also have presented a series of opacities from transition metals of interest to astrophysics. Our calculations appear more consistent than similar opacities from the OP database [11]. We hope that our new OPLIB opacity tables, which are expected to be available in 2015, will prove useful to astrophysical modeling efforts and other dense plasma studies.

\section{Acknowledgments}

The Los Alamos National Laboratory is operated by Los Alamos National Security, LLC for the National Nuclear Security Administration of the U.S. Department of Energy under Contract No. DE-AC52-06NA25396.

\section{References}

[1] N. H. Magee et al, Astronomical Society of the Pacific Conference Series 78, 51 (1995). 
[2] P. Hakel and D. P. Kilcrease, 14th Topical Conference on Atomic Processes in Plasmas, Eds: J. S. Cohen, S. Mazevet, and D. P. Kilcrease, (New York: AIP), pp 190-202 (2004).

[3] D.P. Kilcrease et al, High Energy Density Phys. XX, YYYY (2015).

[4] N. H. Magee et al, 14th Topical Conference on Atomic Processes in Plasmas, Eds: J. S. Cohen, S. Mazevet, and D. P. Kilcrease, (New York: AIP), pp 168-179 (2004).

[5] P. Hakel et al, J. Quant. Spectrosc. Rad. Transfer 99, 265 (2006).

[6] J. Colgan, D.P. Kilcrease, N.H. Magee, Jr., G.S.J. Armstrong, J. Abdallah, Jr., M E. Sherrill, C.J. Fontes, H.L. Zhang, and P. Hakel, Proceedings of the International Conference on Atomic and Molecular Data, pp 17 (2013).

[7] J. Colgan, D.P. Kilcrease, N.H. Magee, Jr., G.S.J. Armstrong, J. Abdallah, Jr., M.E. Sherrill, C.J. Fontes, H.L. Zhang, and P. Hakel, High Energy Density Phys. 9, 369 (2013).

[8] M.J. Seaton, Y. Yan, D. Mihalas, and A.K. Pradhan, Mon. Not. R. Astron. Soc. 266, 805 (1994).

[9] M.J. Seaton and N.R. Badnell, Mon. Not. R. Astron. Soc. 354, 457 (2004).

[10] N.R. Badnell et al, Mon. Not. R. Astron. Soc. 360, 458 (2005).

[11] http://cdsweb.u-strasbg.fr/topbase/testop/TheOP.html

[12] F.J. Rogers and C.A. Iglesias, Ap. JS $\mathbf{7 9}, \quad 507 \quad$ (1992); http://opalopacity.llnl.gov/.

[13] J. Daszyńska-Daszkiewicz and P. Walczak, Mon. Not. R. Astron. Soc. 398, 1961 (2009).

[14] J. Daszyńska-Daszkiewicz and P. Walczak, Mon. Not. R. Astron. Soc. 403, 496 (2010).

[15] J. Daszyńska-Daszkiewicz, W. Szewczuk, and P. Walczak, Mon. Not. R. Astron. Soc. 431, 3396 (2013).

[16] H. Cugier, A \& A 42, 547 (2012).

[17] C. Blancard, P. Cossé, and G. Faussurier, Ap. J. 745, 10 (2012).

[18] S. Turck-Chiéze, D. Gilles, M. Le Pennec, T. Blenski, F. Thais, S. BastianiCeccotti, C. Blancard, M. Busquet, T. Caillaud, J. Colgan, P. Cossè, F. Delahaye, J.E. Ducret, G. Faussurier, C.J. Fontes, F. Gilleron, J. Guzik, J.W. Harris, D.P. Kilcrease, G. Loisel, N.H. Magee, J.C. Pain, C. Reverdin, V. Silvert, B. Villette, and C.J. Zeippen, High Energy Density Phys. 9, 473 (2013).

[19] A.A. Ovechkin, P.A. Loboda, V.G. Novikov, A.S. Grushin, and A.D. Solomyannaya, High Energy Density Phys. 13, 20 (2014).

[20] http://aphysics2.lanl.gov/opacity/lanl 
[21] J.E. Bailey et al, Nature 57, 516 (2015).

[22] S. Turck-Chiéze et al, in preparation (2014).

[23] A. Y. Potekhin and G. Chabrier, Phys. Rev. E 62, 8554 (2000).

[24] J. Abdallah, Jr., R.E.H. Clark and R.D. Cowan, Los Alamos Manual LA-11436M, Vol. I, 1988.

[25] R.D. Cowan, The Theory of Atomic Structure and Spectra (University of California Press, Berkeley, 1981).

[26] R.E.H. Clark, J. Abdallah Jr, and J.B. Mann, Ap. J. 381, 597 (1991); J. Abdallah Jr, H.L. Zhang, C.J. Fontes, D.P. Kilcrease, and B. J. Archer, Journal of Quantitative Spectroscopy \& Radiative Transfer 71, 107 (2001).

[27] S. Mazevet and J. Abdallah, Jr., J. Phys. B 39, 3419 (2006).

[28] J.E. Bailey et al, Phys. Rev. Letts. 99, 265002 (2006).

[29] S.J. Davidson, J.M. Foster, C.C. Smith, K.A. Warburton, and S.J. Rose, Appl. Phys. Letters 52, 847 (1988).

[30] T.S. Perry et al, Phys. Rev. Letts. 67, 3784 (1991).

[31] P.T. Springer et al, Phys. Rev. Letts. 69, 3735 (1992); J. Quant. Spectrosc. Radiat. Transfer 51, 371 (1994).

[32] G. Winhart, K. Eidmann, C.A. Iglesias, and A. Bar-Shalom, Phys. Rev. E 53, R1332 (1996).

[33] J. Abdallah, Jr. and R.E.H. Clark, J. Appl. Phys. 69, 23 (1991).

[34] L.B. DaSilva, B.J. MacGowan, D.R. Kania, B.A. Hammel, C.A. Back, E. Hsieh, R. Doyas, C.A. Iglesias, F.J. Rogers, and R.W. Lee, Phys. Rev. Letts. 69, 438 (1992).

[35] J. Zhang, H. Li, Y. Zhao, G. Xiong, Z. Yuan, H. Zhang, G. Yang, J. Yang, S. Liu, S. Jiang, Y. Ding, B. Zhang, Z. Zheng, Y. Xu, X. Meng, and J. Yan, Phys. of Plasmas 19, 113302 (2012). 